\title{
Identification of molecular markers for pre-thrombotic state: validation in the rabbits with tibia fracture or lung cancer
}

\author{
Yan $\mathrm{Qi}^{1,2}$, Xin $\mathrm{Hu}^{3}$, Jing Chen ${ }^{1}$, Qian $\mathrm{Wu}^{1}$, Xiaobin Ying ${ }^{1}$, Yan Shi ${ }^{1}$ \\ ${ }^{1}$ Department of Nursing, Shanghai Tenth People's Hospital, School of Medicine, Tongji University, Shanghai 215006, China; ${ }^{2}$ School of Medicine, \\ Jinggangshan University, Ji'an 343009, China; ${ }^{3}$ Department of Neurosurgery, Ji'an Central Hospital, Ji'an 343000, China; \\ Contributions: (I) Conception and design: Y Qi, X Hu, Y Shi; (II) Administrative support: None; (III) Provision of study materials or patients: None; \\ (IV) Collection and assembly of data: Y Qi, X Hu, J Chen, Q Wu, X Ying; (V) Data analysis and interpretation: Y Qi, X Hu, J Chen; (VI) Manuscript \\ writing: All authors; (VII) Final approval of manuscript: All authors. \\ Correspondence to: Yan Shi, RN, Professor. No. 301, Yanchang Middle Road, Shanghai 215006, China. Email: nih0325@163.com.
}

Background: Currently there is no reports on identifying molecular markers for pre-thrombotic state in animal experimental settings. It's necessary to explore the potential molecular markers for pre-thrombotic state to provide evidence for the pharmacotherapy of venous thromboembolism (VTE).

Methods: A total of 36 male rabbits were randomly divided into Sham, Tibia fracture, Lung cancer, Tibia fracture and lung cancer group. Pro-thrombin time (PT), thrombin time (TT), activated partial pro-thrombin time (APTT), fibrinogen (FIB), D-dimer (D-D) and anti-thrombin III (AT-III) at baseline, $1,7,14$ and 21 days after operation were detected. Immuno-histochemical staining of tumor tissue sections was performed to observe the expression of CD31, VEGF and vWF. Western blot and qPCR were used to detect the expression levels of ANG and VEGF gene in tumor tissues. Furthermore, we analyzed the tumor tissue with HE staining, immunochemistry, qPCR and Western blot. all the data were statistically analyzed with GraphPad Prism 6.

Results: There were 0, 1, 2, 4 cases of VTE in sham, tibia fracture, lung cancer, tibia fracture and lung cancer group respectively. The level of PT, APTT, TT and AT-III were significantly decreased after operation (all $\mathrm{P}<0.05$ ), whereas the level of FIB and D-D were significantly increased after operation (all $\mathrm{P}<0.05)$. The level of PT, APTT, TT and AT-III were gradually decreased in tibia fracture, lung cancer, tibia fracture and lung cancer group respectively (all $\mathrm{P}<0.05$ ), while the level of FIB and D-D were gradually increased in tibia fracture, lung cancer, tibia fracture and lung cancer group respectively (all $\mathrm{P}<0.05$ ). The medullary lymphocytes increased significantly in the lung cancer group, tibia fracture and lung cancer group (all $\mathrm{P}<0.05$ ), and the number of medullary lymphocytes in tibia fracture and lung cancer group is significantly higher than that of lung cancer group $(\mathrm{P}<0.001)$. The expression of CD31, VEGF and vWF in Tibia fracture and lung cancer group is significantly higher than that of lung cancer group (all $\mathrm{P}<0.001$ ). The expression ANG and VEGF in tibia fracture and lung cancer group is significantly higher than that of lung cancer group (all $\mathrm{P}<0.01)$.

Conclusions: PT, TT, APTT, FIB, D-D and AT-III seem to be sensitive molecular markers for identifying the pre-thrombotic state. CD31, VEGF, ANG and vWF play an important role in the progression of the pre-thrombotic state.

Keywords: Venous thromboembolism (VTE); fracture; lung cancer; pre-thrombotic state; molecular marker

Submitted Jul 18, 2019. Accepted for publication Sep 12, 2019.

doi: $10.21037 /$ tcr.2019.09.44

View this article at: http://dx.doi.org/10.21037/tcr.2019.09.44 


\section{Introduction}

Venous thromboembolism (VTE) is a very common disease in clinical setting, the prophylaxis of VTE is on the top agenda of current related researches (1). It's been found that the blood tend to be hyper-coagulable before thromboembolic disease such as VTE (2). Pre-thrombotic state refers to the imbalance of coagulation, anticoagulation, and fibrinolysis mechanisms. Namely, the pathological conditions favoring thrombosis caused by functional changes in vascular endothelial cells, platelets, coagulation, anticoagulation and fibrinolysis systems (3). It's been reported that the pre-thrombotic state is highly associated with the occurrence of VTE, whereas the pre-thrombotic state is attributed to several risk factors, such as malignant tumor and fracture $(4,5)$. Therefore, the early identification of patients with potential risk of pre-thrombotic state are essential to the progress of VTE.

Currently there is no well-recognized diagnostic criteria for pre-thrombotic state (6). Pro-thrombin time (PT), activated partial pro-thrombin time (APTT), D-dimer (D-D) etc. are clinically used diagnostic indicators for prethrombotic state. Based on literature review, we have found that currently most related studies are conducted in human beings, and the design is generally retrospective, significant bias can be existed with consideration to the individuality and heterogeneity of investigated subjects. Besides, there is no reports on identifying molecular markers for prethrombotic state in animal experimental setting. Therefore, we attempted to identify the molecular markers for prethrombotic state in animal experiments to provide insights into the prophylaxis of VTE.

\section{Methods}

\section{Animals}

The animal experimental protocols were approved by the Animal Care and Use Committee of Shanghai Tenth People's Hospital (SHSY-IEC-KY-4.0/17-46/01) and complied with the ARRIVE guidelines. In this experiment, 36 New Zealand white rabbits were used, and it were provided by the Central Laboratory of the tenth Hospital of Shanghai. All rabbits were male. The age of rabbits ranged from 92 to 120 days with an average age of $104 \pm 6.3$ days, and the average body weight was $2.1 \pm 0.3 \mathrm{~kg}$. All rabbits were kept in a condition of no specific pathogens (SPF).

\section{Experimental design}

A total of 36 New Zealand male rabbits were randomly distributed to sham, tibia fracture, lung cancer, tibia fracture and lung cancer group with nine rabbits for each group. The sham group received no surgery intervention, the tibia fracture group received tibia fracture intervention, the lung cancer group received VX2 lung cancer interjection, while the tibia fracture and lung cancer group received tibia fracture intervention and VX2 lung cancer interjection at the same time. All animals received chloral hydrate intraperitoneal injection (Chloral hydrate, $0.3 \mathrm{~g} / \mathrm{kg}$, Hesheng, China, soluble in $0.9 \%$ saline solution) under the instruction for anesthesia in rabbits prior to surgery (7). All the rabbits were sacrificed after 21 days of operation. We observed and detected the PT, thrombin time (TT), APTT, fibrinogen (FIB), D-D and anti-thrombin III (AT-III) at baseline, 1, 7, 14 and 21 days after operation. Furthermore, we analyzed the VX2 tumor tissue with HE staining, immunochemistry, qPCR and Western blot (Figure 1). With all those resorts, we attempted to explore the molecular markers for prethrombotic state.

\section{The models of rabbits with tibia fracture or lung cancer}

The tibia fracture rabbit model was established with following steps: we habituated animals to handling before beginning anesthesia. After chloral hydrate (chloral hydrate, $0.3 \mathrm{~g} / \mathrm{kg}$, Hesheng, China, soluble in $0.9 \%$ saline solution) intraperitoneally injection, the left forelimb of the rabbit was shaved, the skin was cut along the lateral side of the middle segment, and the humeral muscles and fascia were bluntly separated to expose the bone (Figure 2A). Furthermore, we used surgical wire saws and hemostats to gradually increase the force to cause transverse humeral fractures. Then the last layer wounds were sutured, and the wounds were aseptically treated with penicillin and it was properly wrapped and reared after operation. The recovery of wound was regularly observed.

The rabbit model with VX2 lung cancer was established with following steps: the tumor-bearing rabbits were obtained from the Central Laboratory of Shanghai Tenth Hospital (Figure 2B). After being sacrificed under sterile conditions, isolation of VX2 tumors was performed with surgical methods (Figure 2C). The tumors were placed in petri dishes with PBS buffer (Figure 2D). Then we used 


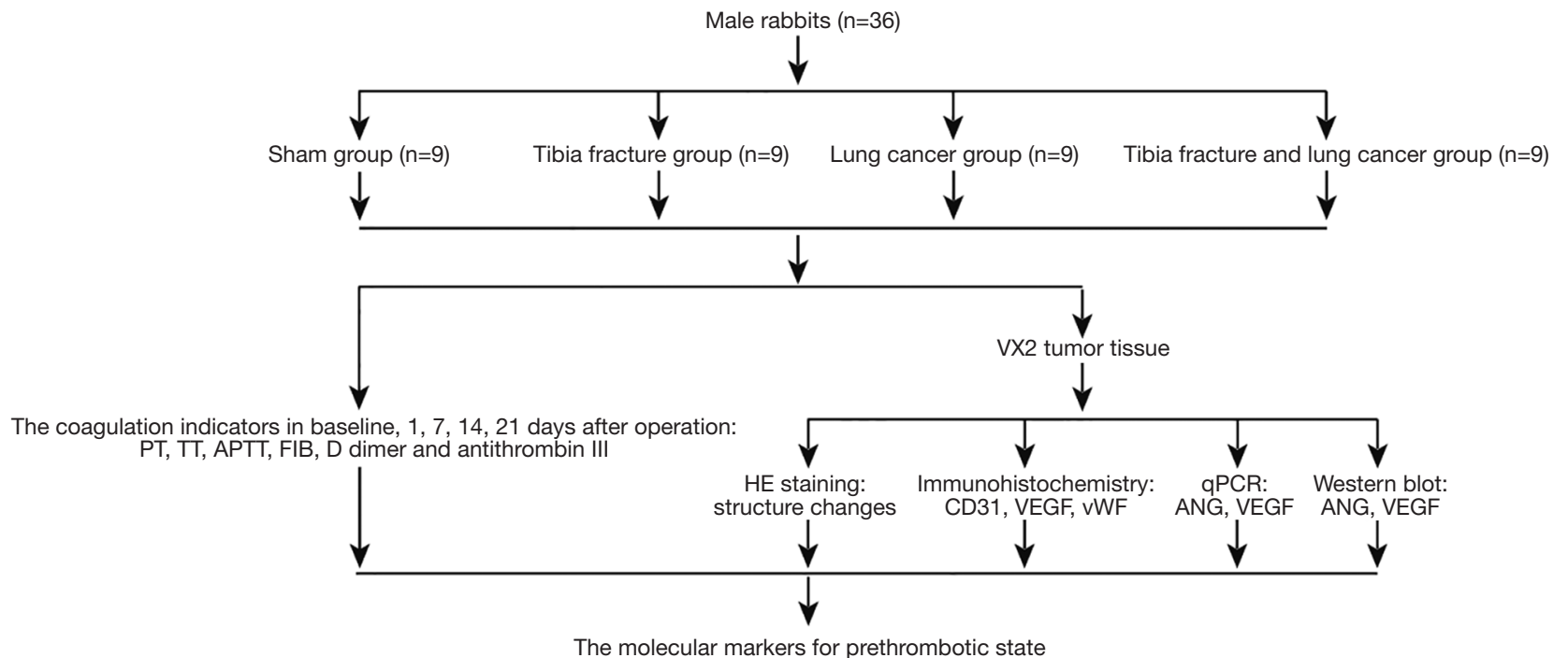

Figure 1 The flow chart of study design. PT, pro-thrombin time; TT, thrombin time; APTT, activated partial pro-thrombin time; FIB, fibrinogen; D-D, D-dimer; AT-III, anti-thrombin III.
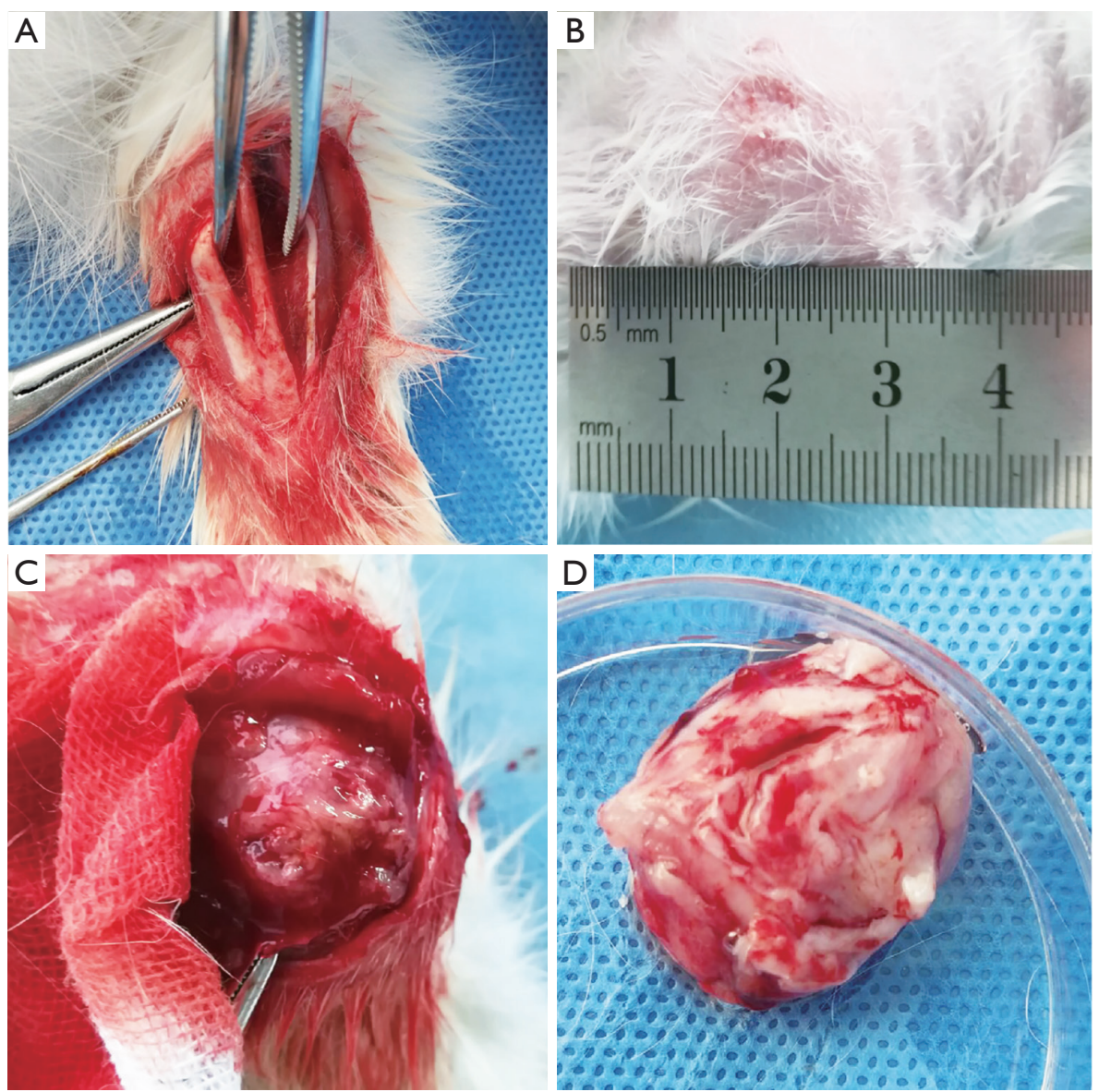

Figure 2 the schematic diagram of modeling. (A) Anatomically exposure of tibia for fracture; (B) the tumor-bearing rabbits; (C) isolation of VX2 tumors; (D) the collection of VX2 tumor. 
ophthalmic scissors to cut the tumor tissue into tissue pieces of about $1 \mathrm{~mm}^{3}$ to prepare a tissue suspension. The tumor-bearing rabbits were anesthetized (chloral hydrate, $0.3 \mathrm{~g} / \mathrm{kg}$, Hesheng, China, soluble in $0.9 \%$ saline solution) and fixed on the operating table. After the alcohol disinfection in the right chest position, the hairs in the chest area were removed. The puncture site was determined under CT machine and aseptically processed. After entering the expected position of the right lower lung under CT guidance, $0.2 \mathrm{~mL}$ of VX2 tumor tissue suspension was injected, and the corresponding imaging examination was performed after needle removal to determine that whether there were complications such as pneumothorax.

\section{Specimen collection and processing}

Local infiltration anesthesia around the area of ear vein with application of local anaesthetic cream (approximately $2 \mathrm{~mL}$, Mingxin, China) was performed when collecting blood from the marginal ear vein of rabbits, and a small amount of apples or carrots were fed to promote the postoperative eating. Since the indexes analyzed in our study were too much, we collected $5 \mathrm{~mL}$ venous blood from the ear vein of every rabbit at the baseline, 1st, 7th, 14th and 21 st day after surgery. And it was used to detect the coagulation index after anticoagulation processing. The high-frequency colored Doppler was used to measure the presence or absence of thrombosis in the limbs of rabbits. Twenty-one days after operation, the weight of the rabbits was averagely $2 \mathrm{~kg}$. The VX2 tumor tissues in lung cancer, tibia fracture and lung cancer group were dissected. And the size, shape and color of the tumor were recorded, and the pathological examination of the formed tumor tissue was performed.

\section{Methods of euthanasia}

The rabbits were anesthetized (chloral hydrate, $0.3 \mathrm{~g} / \mathrm{kg}$, Hesheng, China, soluble in $0.9 \%$ saline solution) and $10 \mathrm{~min}$ later $30 \mathrm{~mL}$ air was injected into the ear margin (8). We confirmed that absence of heart beat for 5 minutes to confirm animal death, then the rabbits' veins were exposed by anatomical methods to observe the formation of deep vein thrombosis. We terminated the painful procedure and gave more fentanyl $(0.02 \mathrm{mg} / \mathrm{kg}$, Qingyu, China) to relieve the pain when significant tremors or movements were observed during the process.

\section{The detection of coagulation parameters}

Two $\mathrm{mL}$ blood was taken from the previously collected blood specimen using a pipette. The blood was centrifuged $(2,000 \times \mathrm{g}, 10 \mathrm{~min})$, and the supernatant was taken. The blood coagulation analyzer (Dexer X980, German) was used to measure the coagulation parameters (PT, TT, APTT, FIB).

The D-D and AT-III were detected as follows: the resting $3 \mathrm{~mL}$ blood of each specimen was centrifuged $(3,000 \times \mathrm{g}$, $30 \mathrm{~min}$ ), and the supernatant was taken. The D-dimer level was determined by ELISA (Abcam ab196269, USA). The AT-III levels were determined by the chromogenic substrate method (Abcam EP5372, USA). All the procedures were carried out in comply with the product instructions.

\section{Hematoxylin and Eosin (HE) staining}

The dissected tumor tissues were fixed with $10 \%$ formaldehyde solution, and a tissue with a size of $1.5 \mathrm{~cm}$ $\times 1.5 \mathrm{~cm}$ and a thickness of $0.3 \mathrm{~cm}$ was taken. The tissue was sliced after paraffin embedding. The further procedures of HE staining were conducted as previously described (9). Finally, the specimen was examined and photo-graphed by light microscope (Olympus OX50) at 40× view field.

\section{Immunohistochemistry}

The tumor tissue sections were subjected to immunehistochemical staining to observe the expression of CD31, VEGF and vWF. First, the obtained paraffin section is dewaxed to water using xylene, ethanol, distilled water etc. The tissue sections were immersed in a citrate buffer (purified to boiling) at $\mathrm{pH}$ 6.0, and then heated in a medium-range microwave for 10 minutes, followed by washing with distilled water and PBS. The endogenous peroxidase activity was eliminated by $3 \% \mathrm{H}_{2} \mathrm{O}_{2}$. Then rinsed with distilled water, and immersed in PBS twice for $5 \mathrm{~min}$ each time. After removing the PBS, the appropriate primary dilutions of the corresponding primary antibody were added to the tissues for $2 \mathrm{~h}$ (incubation at room temperature). After incubation, the sections were washed with PBS 3 times (5 min per time), pour PBS, add EnVision reagent (secondary antibody) for $30 \mathrm{~min}$, and it was washed with PBS 3 times after the reaction, and added DAB for $10 \mathrm{~min}$. Finally, the specimens were observed under microscope (Olympus OX50). 
Table 1 The prime sequence of real time fluorescent quantitative PCR

\begin{tabular}{lll}
\hline \multirow{2}{*}{ Prime } & \multicolumn{1}{c}{ Sequence $\left(5^{\prime}-3^{\prime}\right)$} & R \\
\cline { 2 - 3 } & \multicolumn{1}{c}{$\mathrm{F}$} & CGGGATCCTTACGGACGACGGAAAATTGA \\
ANG & CGGAATTCATGCAGGATAACTCCAGGTACAC & AAAATGGCGAATCCAATTCC \\
GAPDH & CGGGAACCAGATCTCTCACC & GGCTGTTGTCATACTTCTCATGG \\
\hline
\end{tabular}

\section{Western blotting}

The collected tumor tissue was cut with scissors, then the homogenate with PMSF was lysed for $30 \mathrm{~min}$. And then transferred to a centrifuge tube for centrifugation $(12,000 \times \mathrm{g}, 5 \mathrm{~min})$ at $4{ }^{\circ} \mathrm{C}$, the supernatant was stored at $-80{ }^{\circ} \mathrm{C}$ for Western blotting. The protein concentration was measured by the bicinchoninic acid (BCA) method with enhanced BCA protein assay kit (Beyotime, China). Samples were boiled at $100{ }^{\circ} \mathrm{C}$ for $10 \mathrm{~min}$ in sample buffer. Equal amounts of protein $(50 \mu \mathrm{g}$ per sample) were separated by electrophoresis on $7.5-12 \%$ Tris-glycine sodium dodecyl sulfate polyacrylamide gels. After electrophoresis, the proteins were transferred onto nitrocellulose membranes that were blocked and incubated with anti-ANG $(1: 1,000$, Abcam ab99970) and anti-VEGF (1:1,000, Abcam ab32152, USA) antibodies at $4{ }^{\circ} \mathrm{C}$ overnight. After washing, the membranes were incubated with corresponding secondary antibodies (1:3,000, Abcam ab150077, USA) for 4 hours at a temperature of $23-25^{\circ} \mathrm{C}$. After being rinsed, the membranes were reacted with enhanced chemiluminescence detection reagents (Mingo 1207, Shanghai). Then the protein bands were scanned and analyzed on PhosphorImager (Tianneng, Shanghai). The relative quantity of proteins was analyzed using Image $J$ and it normalized to that of loading controls [GAPDH (Abcam ab181602, USA)].

\section{qPCR detection}

The TRIzol lysate was added into the tumor tissue, after the lysis was completed, the cells were collected in a centrifuge tube and added with appropriate amount of chloroform. After centrifugation at $12,000 \times \mathrm{g}$ for $15 \mathrm{~min}$ at $4{ }^{\circ} \mathrm{C}$, the supernatant was taken and added with isopropanol. Then centrifuged the specimen again for $10 \mathrm{~min}$, and the supernatant was discarded and the precipitate was collected. Furthermore, rinsing the precipitate with $75 \%$ alcohol. Besides, we added the enzyme-free water to dissolve the RNA for the concentration measurement of RNA.
The sample concentration was uniformly calibrated to $50 \mathrm{ng} / \mathrm{\mu L}$ according to the measurement result. Finally, the reverse transcription syntheses of cDNA were performed after adding the reagents under the instruction of Takara PrimeScript RT reagent Kit (RR037A). The gene primer sequences required for the experiment were presented in Table 1.

\section{Statistical analysis}

The statistical data in this present study were presented as mean \pm standard deviation $(\overline{\mathrm{x}} \pm \mathrm{s})$. Graph Pad Prism 6 (GraphPad Software, San Diego, CA) was used for all statistical analysis and Chart production. The Student's $t$-test was used for the comparison of single two groups. The comparison between multiplied groups was analyzed by ANOVA, and the Bonferroni method was used for further comparison of two groups. A value of $\mathrm{P}<0.05$ was considered as being statistically significant.

\section{Results}

\section{The VTE incidence}

As Figure 3 presented, there were 0, 1, 2, 4 cases of VTE in sham, tibia fracture, lung cancer, tibia fracture and lung cancer group respectively, and the difference on the incidence of VTE among those groups were statistically significant $(\mathrm{P}=0.037)$

\section{The changes of PT, TT, APTT, FIB, D-D and AT-III level}

As Figure 4 showed, the baseline PT, TT, APTT, FIB, $\mathrm{D}-\mathrm{D}$ and AT-III levels were similar in each group, and the difference was not statistically significant $(\mathrm{P}>0.05)$. Significantly changes were detected in the level of PT, TT, APTT, FIB, D-D and AT-III in baseline, day 1, 7,14 and 21 among different group (all $\mathrm{P}=0.009$ ), but not in Sham group $(\mathrm{P}=0.071)$, the level of PT, APTT, TT and AT-III were 


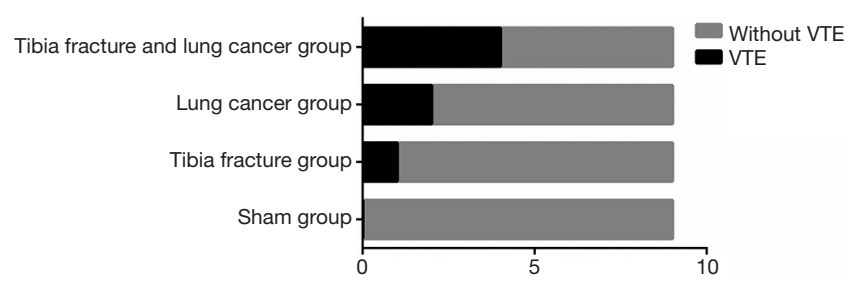

Figure 3 The VTE incidence of different groups. VTE, venous thromboembolism.

significantly decreased after operation (all $\mathrm{P}<0.05$ ), whereas the level of FIB and D-D were significantly increased after operation (all $\mathrm{P}<0.05$ ).

Furthermore, significantly changes were detected in the level of PT, TT, APTT, FIB, D-D and AT-III among tibia fracture, lung cancer, tibia fracture and lung cancer group (all $\mathrm{P}<0.05$ ). The level of PT, APTT, TT and AT-III were gradually decreased in tibia fracture, lung cancer, tibia fracture and lung cancer group respectively (all $\mathrm{P}<0.05$ ). While the level of FIB and D-D were gradually increased in tibia fracture, lung cancer, tibia fracture and lung cancer group respectively (all $\mathrm{P}<0.05)$.

\section{HE staining}

As Figure $5 A$ showed, for sham group, the lung tissue was closely arranged and aggregated, but for lung cancer group, tibia fracture and lung cancer group. The tissues were in heterogeneous state with large morphological abnormalities, the nucleus of the tumor cells is deeply stained and some of the mitotic figures are visible. As Figure $5 B$ showed, the medullary lymphocytes increased significantly in the lung cancer group, tibia fracture and lung cancer group (all $\mathrm{P}<0.05$ ), and the number of medullary lymphocytes in tibia fracture and lung cancer group is significantly higher than that of lung cancer group $(\mathrm{P}<0.001)$.

\section{Immunobistochemistry on the expression of CD31, VEGF and $v W F$}

As Figure 6 indicated, there were significant differences on the expression of CD31, VEGF and vWF among sham group, lung cancer group, tibia fracture and lung cancer group (all $\mathrm{P}<0.001$ ). The expression of CD31, VEGF and $\mathrm{vWF}$ in tibia fracture and lung cancer group is significantly higher than that of lung cancer group (all $\mathrm{P}<0.001$ ).

\section{The expression level of ANG and VEGF}

As Figure 7 showed, there were significant differences on the expression of ANG and VEGF mRNA among sham group, lung cancer group, tibia fracture and lung cancer group (all $\mathrm{P}<0.001$ ). The expression of $\mathrm{ANG}$ and $\mathrm{VEGF}$ in tibia fracture and lung cancer group is significantly higher than that of lung cancer group (all $\mathrm{P}<0.01$ ).

\section{Discussion}

VTE is an important complication around perioperative period with high clinical morbidity and great risk, the prophylaxis of VTE is essential to the patients' prognosis $(10,11)$. It's been reported that the incidence VTE in adult population is over $0.1 \%$, and the incidence rate is $0.5 \%$ in the elderly over 80 years old, and there are more than 2 million new cases of VTE every year $(12,13)$. Besides, the reports (14-16) from different countries have showed that the incidence of VTE is increasing year by year. Therefore, VTE is currently a research hotspot in the global medical community. To date, it's well-believed that the prethrombotic state is an essential step to the occurrence of VTE, and the patients with surgery, trauma, and cancer have a higher risk of pre-thrombotic state, and these highrisk factors are inextricably linked to the progress of VTE $(17,18)$. However, most related studies are focused on clinical setting, and most studies design are retrospective, significant bias can exist. It's necessary to evaluate the prethrombotic state from the perspectives of animal experiments. Besides, once the VTE (particularly the pulmonary embolism) occurs, the clinical mortality rate is extremely high. It's been reported that about 20,000 people die of pulmonary embolism each year in USA (19). Therefore, it's urgently necessary to find clues in these high-risk factors leading to VTE. Using various indicators and inflammation and signal factor changes are needed to timely determine the risk of VTE, to further identify the mechanism of VTE for early intervention to prevent the occurrence of VTE. This present study aimed to use animal models to simulate the high-risk factors which is commonly-see in clinical settings, adopt scientific methods to control the high-risk factors within a relatively uniform and controllable range, and collect different indicators for results analysis. Based on the results, we have found that the fracture and cancer are two important risk factors of pre-thrombotic state, the PT, TT, APTT, FIB, D-D and AT-III can be used as good 

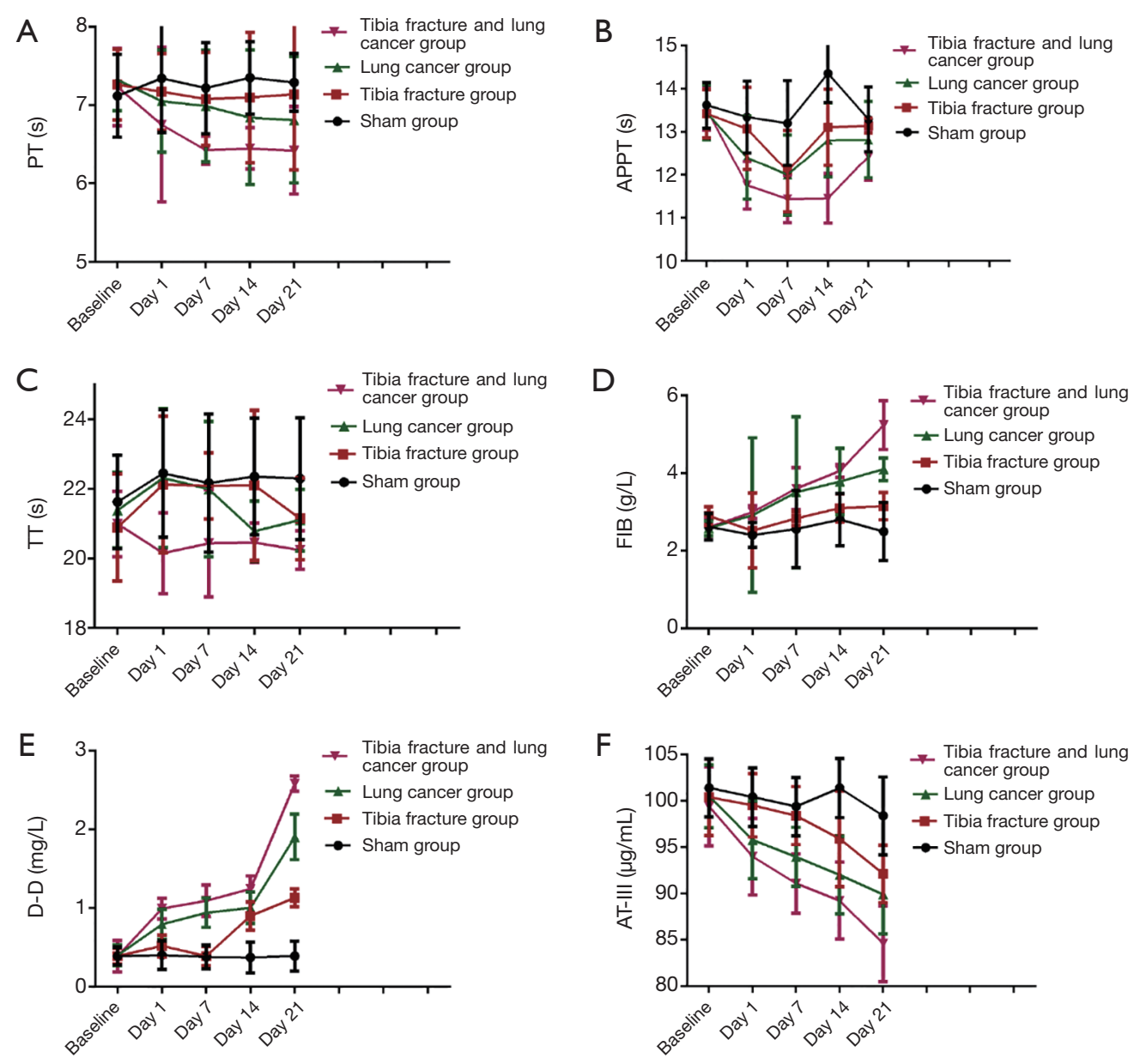

Figure 4 The chart on the changes of PT, APTT, TT, FIB, D-D and AT-III level. (A) The flow chart of PT changes; (B) the flow chart of APTT changes; (C) the flow chart of TT changes; (D) the flow chart of FIB changes; (E) the flow chart of D-D changes; (F) the flow chart of AT-III changes. PT, pro-thrombin time; TT, thrombin time; APTT, activated partial pro-thrombin time; FIB, fibrinogen; D-D, D-dimer; AT-III, anti-thrombin III.

molecular markers of pre-thrombotic state of VTE, besides, the VEGF and vWF play considerable roles in the progress of pre-thrombotic state.

At present, the PT, APTT and TT are frequently used clinically to analyze the coagulation function, the results of this present study indicate that all the PT, TT, APTT, FIB, D-D and AT-III are the sensitive indicators of coagulation function. Previous study has referred that TT can better reflect the process of transforming FIB into fibrin by thrombin in vivo, it may be a better indicator for the risk of VTE (20). D-D is a specific molecular marker that can reflect the hyper-coagulable state and fibrinolysis activation of the body (21). It is extremely low in normal condition. Meanwhile, FIB is an acute phase reaction protein and an important marker of coagulation activation (22). According to the results, there was a sharp increase in FIB and D-D on the first postoperative day after modeling, and during the gradual recovery process, the FIB and D-D showed a downward trend. It can be explained that under the stimulation of surgical stress and trauma, the blood coagulation system in the body activates, and then promote the progress of secondary fibrinolysis. Additionally, the lung cancer group, tibia fracture and lung cancer group showed a gradual increase with the change of postoperative time, it can be explained that under the stimulation of VX2 lung cancer, the D-D and FIB are continuously stimulated, thereby leading to pro-thrombotic state. It's been reported that the tumor cells can destroy the normal tissues and 


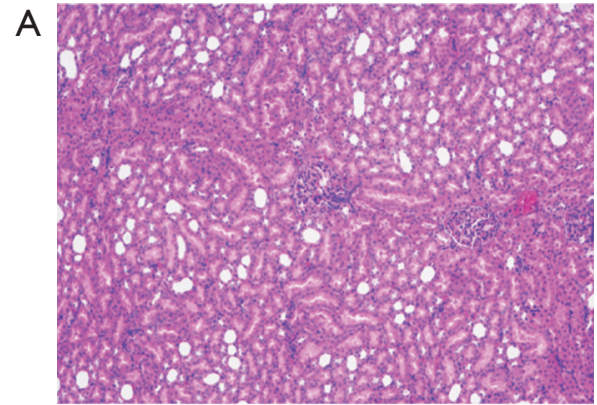

Sham group

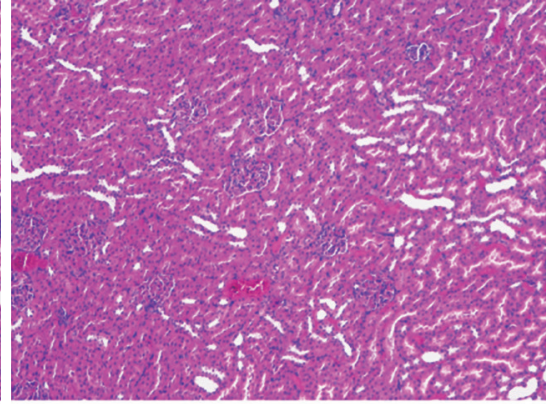

Lung cancer group

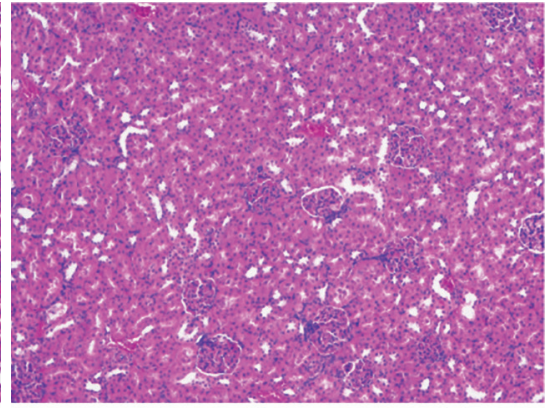

Tibia fracture and lung cancer group

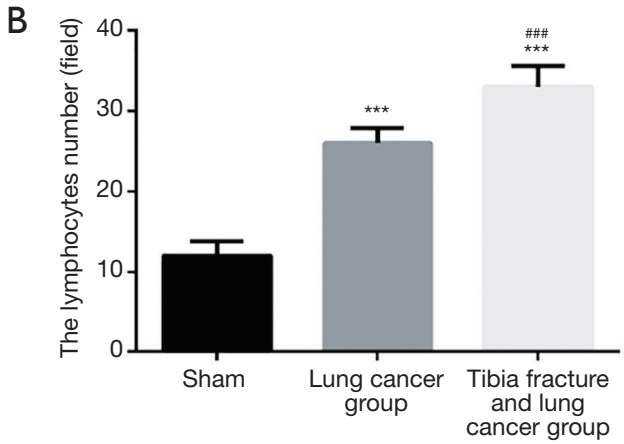

Figure 5 The results of HE staining. (A) The HE staining of lung tissue ( $\times 40)$; (B) the statistical graph on the number of lymphocytes (***, $\mathrm{P}<0.001$ vs. the sham group; ${ }^{\# \# \#}, \mathrm{P}<0.001$ vs. the lung cancer group).

stimulate the activation of the coagulation system through an exogenous pathway, which in turn leads to a consistent increase of FIB and D-D levels $(23,24)$. At the same time, several studies (25-27) have pointed out that AT-III acts as an important serine protease inhibitor in the body's anticoagulant regulation, and serine protease is inactivated when the AT-III binds to a large number of active coagulation factors under the hyper-coagulable condition $(28,29)$. Therefore, the change of FIB, D-D and AT-III may be early signs of pre-thrombotic state.

The role of CD31, VEGF, ANG and $\mathrm{vWF}$ must be considered. The expression of CD31, VEGF, ANG and vWF in Tibia fracture and lung cancer group is significantly higher than that of Lung cancer group, which is consistent with the findings of previously published studies (30-32). The reason may be that VEGF stimulates neovascularization regardless of fracture or tumor (33). Particularly, it is pointed out that tumor cells have a large oxygen demand, which often causes hypoxia to stimulate the body to secrete VEGF, and VEGF is currently the strongest cytokine that stimulates angiogenesis and enhances vascular permeability (34). The neovascularization caused by VEGF stimulation is prone to rupture damage and activates the coagulation system (35), resulting in hypercoagulability of the blood, while high vascular permeability leads to leakage of the relevant clotting factors, exacerbating the body's fibrinolysis and eventually leading to the occurrence of VTE $(36,37)$. The increase of ANG with pro-angiogenic activity in tibia fracture and lung cancer group further proves that process (38). As the carrier protein of factor VIII, $\mathrm{vWF}$ is positively correlated with the degree of osteogenic reaction of fracture healing (39). Being an important index of tumor angiogenesis, vWF is not only closely related to tumor growth and metastasis, but also reflects the vascular endothelial injury (40). The emboli generated in VTE inevitably damages vascular endothelial cells, which leads to the release of $\mathrm{vWF}$ (41). Therefore, $\mathrm{vWF}$ may be a late molecular marker for prethrombotic state, further investigation on the role of $\mathrm{vWF}$ in prethrombotic state are warranted.

Several limitations of this present study must be considered. Firstly, we only observed several common coagulation indicators, the changes of some other identified potential molecular such as cell-derived microparticles etc. need further investigation (42). Secondly, we only detected the change of CD31, VEGF, ANG and vWF under the condition of fracture and lung cancer. However, the 
A

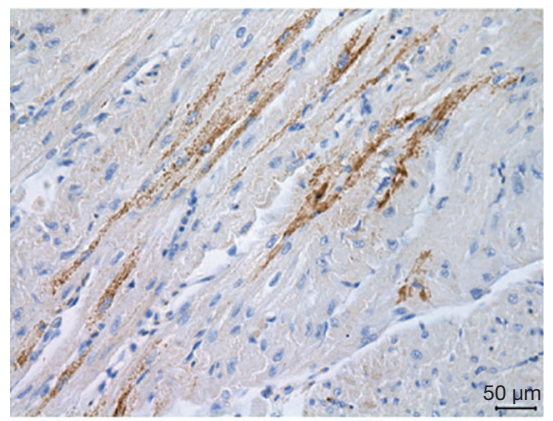

Sham group

B

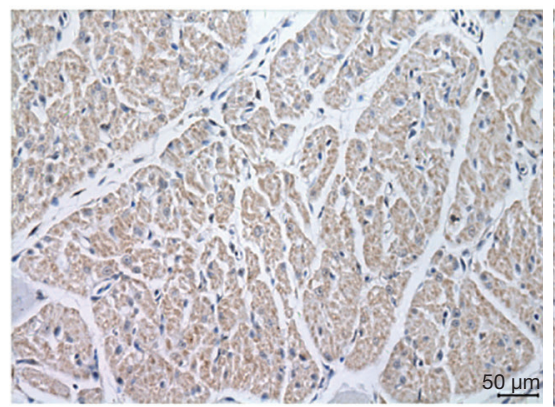

Sham group

C

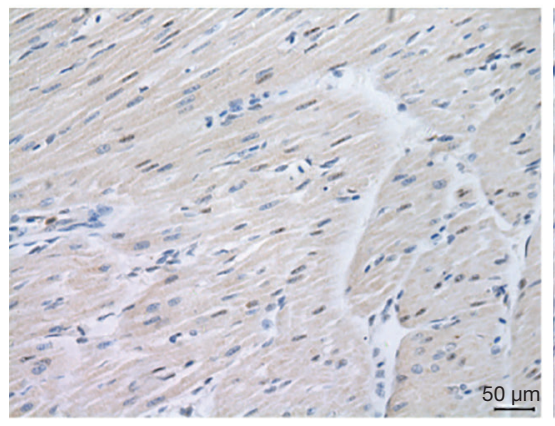

Sham group

D

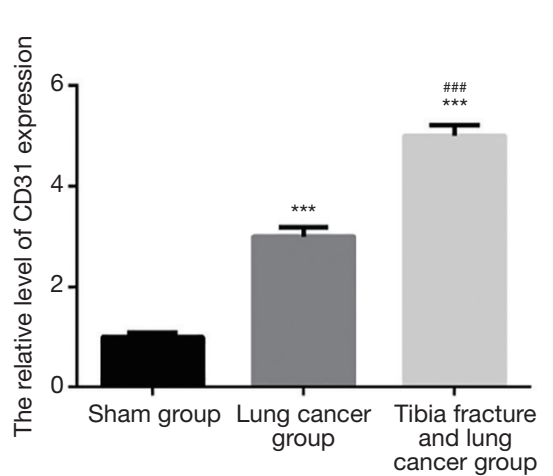

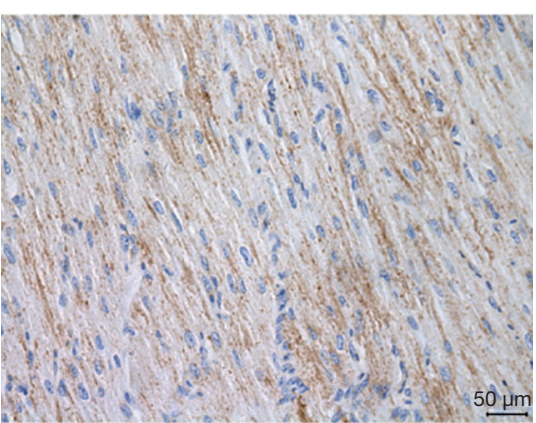

Lung cancer group

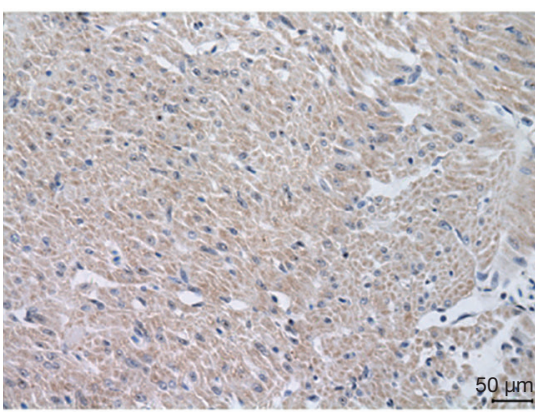

Lung cancer group

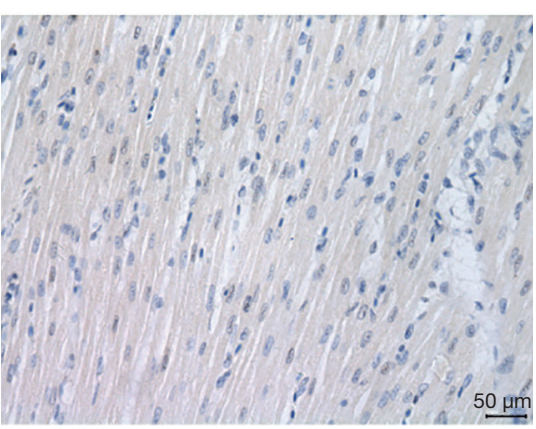

Lung cancer group

$\mathrm{E}$

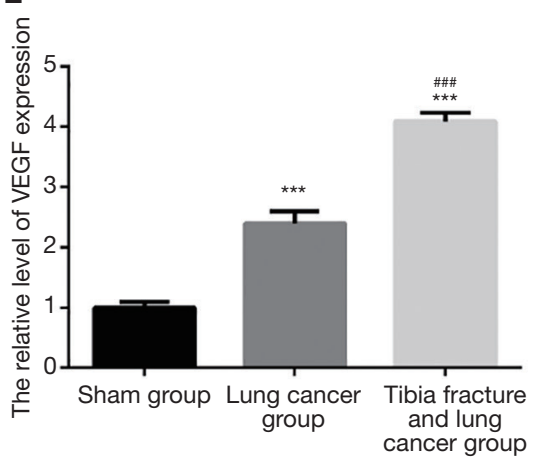

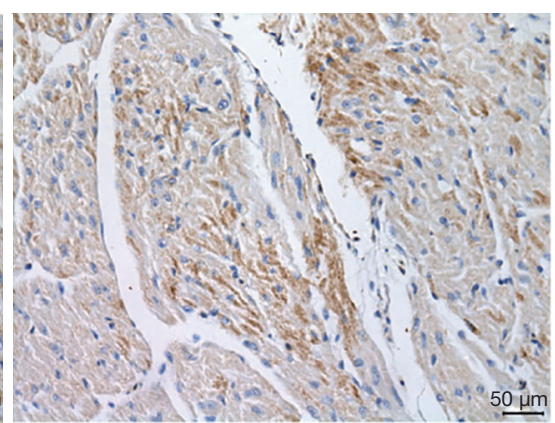

Tibia fracture and lung cancer group

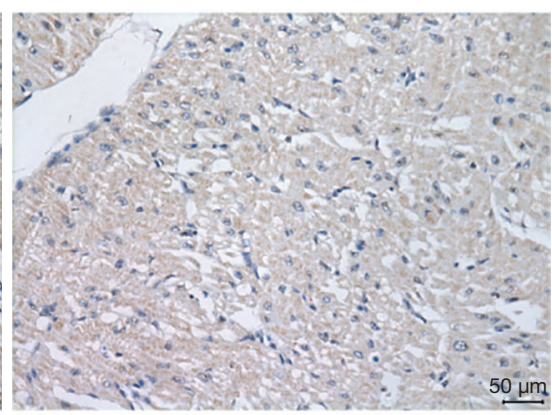

Tibia fracture and lung cancer group

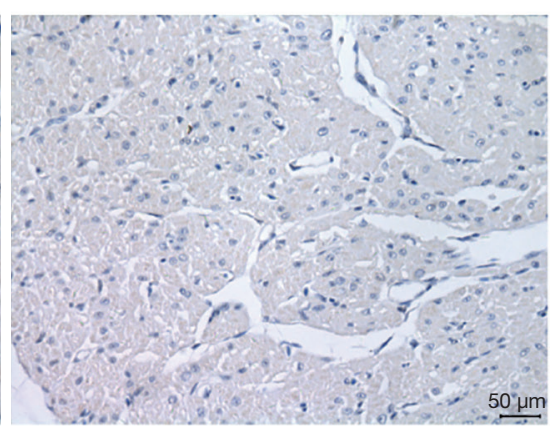

Tibia fracture and lung cancer group

$\mathrm{F}$

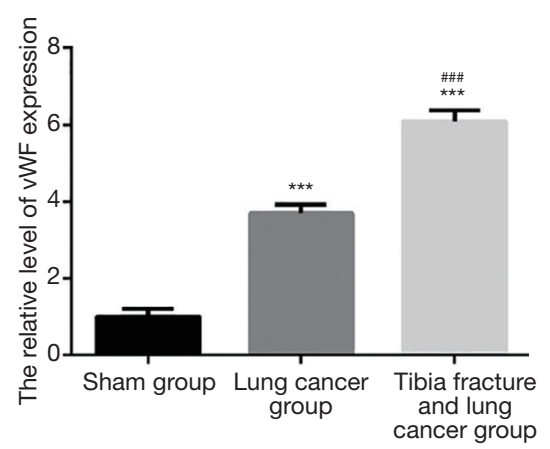

Figure 6 The immunohistochemistry on the expression of CD31, VEGF and vWF. (A) The graph of immunohistochemistry on the expression of CD31; (B) the graph of immunohistochemistry on the expression of VEGF; (C) the graph of immunohistochemistry on the expression of vWF; (D) the statistical graph on the expression of CD31; (E) the statistical graph on the expression of VEGF; (F) the statistical graph on the expression of $\mathrm{vWF}\left({ }^{* * *}, \mathrm{P}<0.001\right.$ vs. the sham group; ${ }^{\# \# \#}, \mathrm{P}<0.001$ vs. the lung cancer group). 

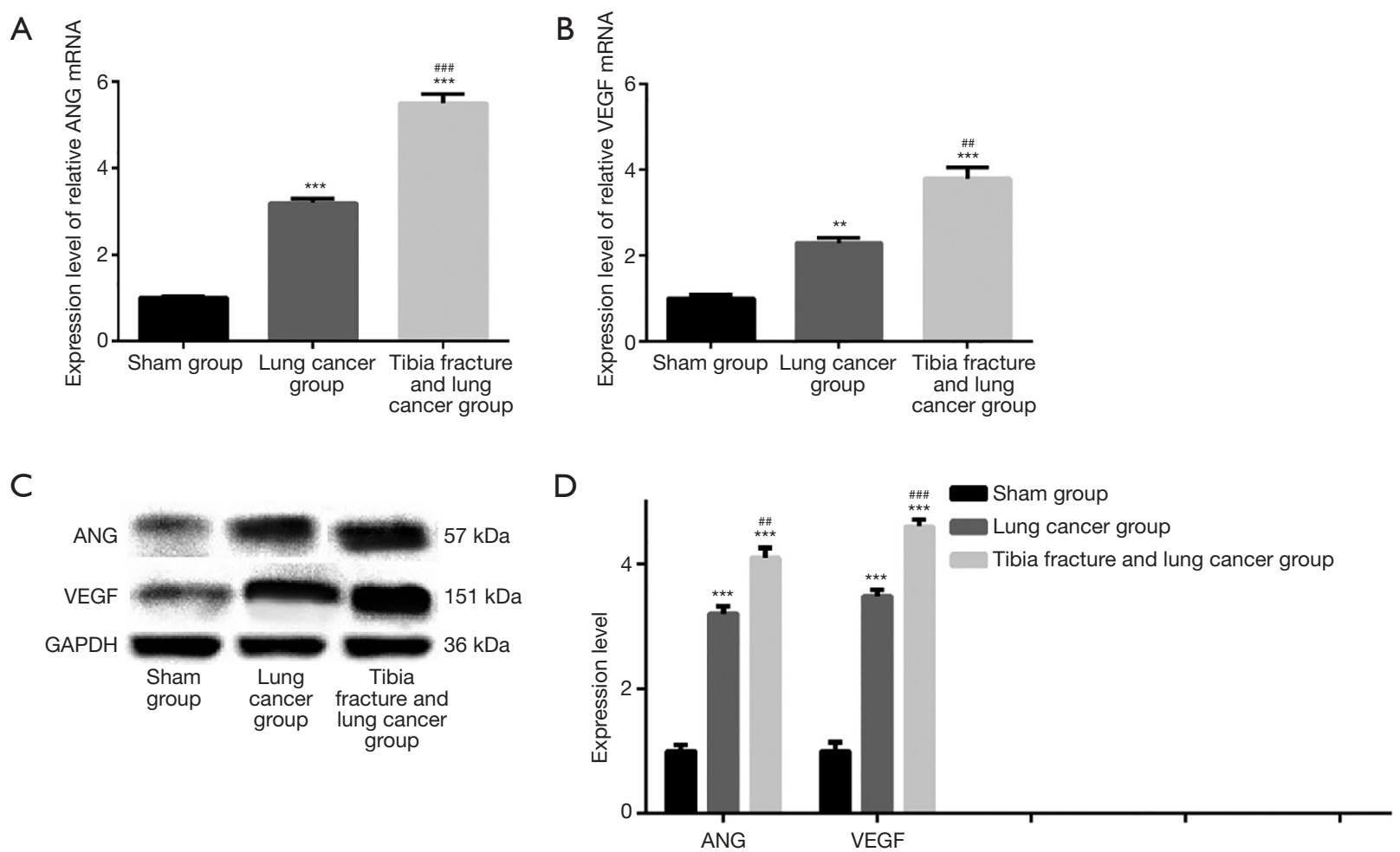

Figure 7 The expression of ANG and VEGF. (A) The graph of qPCR comparison on the expression of ANG; (B) the graph of qPCR comparison on the expression of VEGF; (C) the Western blot graph on the expression of ANG and VEGF; (D) the statistical graph on the Western blot expression of ANG and VEGF (***, $\mathrm{P}<0.001$ vs. the sham group; ${ }^{* *}, \mathrm{P}<0.01$ vs. the sham group; ${ }^{\# \#}, \mathrm{P}<0.001$ vs. the lung cancer group; ${ }^{\# \#}, \mathrm{P}<0.01$ vs. the lung cancer group).

potential mechanisms remain unclear, it's necessary to conduct more studies to identify the related mechanisms to provide more insights into clinical background. Finally, we only identified the potential molecular markers for prethrombotic state in animal experiments, the correspondingly analysis in clinical patients are highlighted in future studies.

\section{Conclusions}

In conclusions, PT, TT, APTT, FIB, D-D and ATIII are good molecular markers for the identification of pre-thrombotic state, and CD31, VEGF, ANG and $\mathrm{vWF}$ play important role in the progress of prethrombotic state. More studies are warranted to elucidate the potential mechanisms of molecular markers in the pre-thrombotic state.

\section{Acknowledgments}

Funding: None.

\section{Footnote}

Conflicts of Interest: The authors have completed the ICMJE uniform disclosure form (available at http://dx.doi. org/10.21037/tcr.2019.09.44). The authors have no conflicts of interest to declare.

Ethical Statement: The authors are accountable for all aspects of the work in ensuring that questions related to the accuracy or integrity of any part of the work are appropriately investigated and resolved. The animal experimental protocols were approved by the Animal Care and Use Committee of Shanghai Tenth People's Hospital (SHSY-IEC-KY-4.0/17-46/01). In compliance with the national or institutional guidelines for the care and use of animals.

Open Access Statement: This is an Open Access article distributed in accordance with the Creative Commons Attribution-NonCommercial-NoDerivs 4.0 International 
License (CC BY-NC-ND 4.0), which permits the noncommercial replication and distribution of the article with the strict proviso that no changes or edits are made and the original work is properly cited (including links to both the formal publication through the relevant DOI and the license). See: https://creativecommons.org/ licenses/by-nc-nd/4.0/.

\section{References}

1. Tritschler T, Aujesky D. Venous thromboembolism in the elderly: A narrative review. Thromb Res 2017;155:140-7.

2. Bartholomew JR. Update on the management of venous thromboembolism. Cleve Clin J Med 2017;84:39-46.

3. Hobbelt AH, Spronk HM, Crijns H, et al. Prethrombotic State in Young Very Low-Risk Patients With Atrial Fibrillation. J Am Coll Cardiol 2017;69:1990-2.

4. Girolami A, Cosi E, Ferrari S, et al. Prethrombotic, prothrombotic, thrombophilic states, hypercoagulable state, thrombophilia etc.: semantics should be respected even in medical papers. J Thromb Thrombolysis 2017;43:390-3.

5. Hawbaker S. Venous thromboembolism in the cancer population: pathology, risk, and prevention. J Adv Pract Oncol 2012;3:23-33.

6. Bai L, Yuan K, Zhao G. The Progress in prethrombotic state of chronic obstructive pulmonary disease. Chin J Gerialr Care 2014;12:92-4.

7. Chen Z, Xiao EH, Kang Z, et al. In vitro and in vivo magnetic resonance imaging with chlorotoxinconjugated superparamagnetic nanoprobes for targeting hepatocarcinoma. Oncol Rep 2016;35:3059-67.

8. She G, Zhou Z, Zha Z, et al. Protective effect of zoledronic acid on articular cartilage and subchondral bone of rabbits with experimental knee osteoarthritis. Exp Ther Med 2017;14:4901-9.

9. Zhang WG, He L, Shi XM, et al. Regulation of transplanted mesenchymal stem cells by the lung progenitor niche in rats with chronic obstructive pulmonary disease. Respir Res 2014;15:33.

10. Ambrosino P, Tarantino L, Di Minno G, et al. The risk of venous thromboembolism in patients with cirrhosis. A systematic review and meta-analysis. Thromb Haemost 2017;117:139-48.

11. Tsikouras P, von Tempelhoff GF, Rath W. Epidemiology, Risk Factors and Risk Stratification of Venous Thromboembolism in Pregnancy and the Puerperium. Z Geburtshilfe Neonatol 2017;221:161-74.
12. Boyle E, Reid J, O’Donnell M, et al. Thromboprophylaxis for varicose vein procedures - A national survey. Phlebology 2019;34:598-603.

13. Buchanan IA, Lin M, Donoho DA, et al. Venous Thromboembolism After Degenerative Spine Surgery: A Nationwide Readmissions Database Analysis. World Neurosurg 2019;125:e165-e174.

14. Song CF, Li H, Tian B, et al. Survey of current status of prevention of venous thromboembolism after thoracic surgery in China. Zhonghua Wai Ke Za Zhi 2017;55:661-6.

15. Imberti D, Barillari G, eXperience VTEIG. RealLife Management of Venous Thromboembolism With Rivaroxaban: Results From EXperience VTE, an Italian Epidemiological Survey. Clin Appl Thromb Hemost 2018;24:241-7.

16. Goecke T, Voigt F, Rath W. Thromboprophylaxis following cesarean section - a nation-wide survey from Germany. J Matern Fetal Neonatal Med 2019. [Epub ahead of print].

17. Falanga A, Russo L, Milesi V, et al. Mechanisms and risk factors of thrombosis in cancer. Crit Rev Oncol Hematol 2017;118:79-83.

18. Branchford BR, Carpenter SL. The Role of Inflammation in Venous Thromboembolism. Front Pediatr 2018;6:142.

19. Essien EO, Rali P, Mathai SC. Pulmonary Embolism. Med Clin North Am 2019;103:549-64.

20. Hu C, Dou G, Sun W, et al. Tissue distribution and excretion of human fibrin glue in New Zealand rabbits. J Pharm Res Int 2018;45:33-6.

21. Li WJ, Sha M, Ma W, et al. Efficacy evaluation of D-dimer and modified criteria in overt and nonovert disseminated intravascular coagulation diagnosis. Int $\mathrm{J}$ Lab Hematol 2016;38:151-9.

22. Zhang GM, Zhang W, Zhang GM. Age-Specific Reference Intervals for PT, aPTT, Fibrinogen and Thrombin Time for Parturient Women. Thromb Haemost 2019;119:894-8.

23. Tinholt M, Sandset PM, Iversen N. Polymorphisms of the coagulation system and risk of cancer. Thromb Res 2016;140 Suppl 1:S49-54.

24. Mitrugno A, Tormoen GW, Kuhn P, et al. The prothrombotic activity of cancer cells in the circulation. Blood Rev 2016;30:11-9.

25. Zhu L, Li C, Wang D. Individual differences of plasma proteins and factors in fresh frozen plasma from Chinese regional blood donors. J Thromb Thrombolysis 2019;47:420-6.

26. Hidaka H, Kokubu S, Sato T, et al. Antithrombin III for 
portal vein thrombosis in patients with liver disease: A randomized, double-blind, controlled trial. Hepatol Res 2018;48:E107-16.

27. Du Z, Shi F, Liu D, et al. Anticoagulant activity of a sulfated Lachnum polysaccharide in mice with a state of hypercoagulability. Bioorg Med Chem Lett 2016;26:5550-6.

28. Chen LB, Meng LL, Chen H, et al. Coagulation and prothrombotic state parameters: clinical analysis in early pregnancy. Zhonghua Yi Xue Za Zhi 2013;93:2146-8.

29. Konanki R, Gulati S, Saxena R, et al. Profile of prothrombotic factors in Indian children with ischemic stroke. J Clin Neurosci 2014;21:1315-8.

30. Lee JM, Kwon HJ, Lai WF, et al. Requirement of Runx3 in pulmonary vasculogenesis. Cell Tissue Res 2014;356:445-9.

31. Whitsett JA, Kalin TV, Xu Y, et al. Building and Regenerating the Lung Cell by Cell. Physiol Rev 2019;99:513-54.

32. Stewart EA, Samaranayake GJ, Browning AC, et al. Comparison of choroidal and retinal endothelial cells: characteristics and response to VEGF isoforms and antiVEGF treatments. Exp Eye Res 2011;93:761-6.

33. Chen J, Fu Y, Day DS, et al. VEGF amplifies transcription through ETS1 acetylation to enable angiogenesis. Nat Commun 2017;8:383.

34. Sargent KM, McFee RM, Spuri Gomes R, et al. Vascular endothelial growth factor A: just one of multiple mechanisms for sex-specific vascular development within the testis? J Endocrinol 2015;227:R31-50.

35. Wu M, Liu Y, Zhang $\mathrm{H}$, et al. Intravenous injection of l-aspartic acid beta-hydroxamate attenuates choroidal

Cite this article as: Qi Y, Hu X, Chen J, Wu Q, Ying X, Shi Y. Identification of molecular markers for pre-thrombotic state: validation in the rabbits with tibia fracture or lung cancer. Transl Cancer Res 2019;8(6):2316-2327. doi: 10.21037/ tcr.2019.09.44 neovascularization via anti-VEGF and anti-inflammation. Exp Eye Res 2019;182:93-100.

36. Hegde PS, Wallin JJ, Mancao C. Predictive markers of anti-VEGF and emerging role of angiogenesis inhibitors as immunotherapeutics. Semin Cancer Biol 2018;52:117-24.

37. Amadio M, Govoni S, Pascale A. Targeting VEGF in eye neovascularization: What's new?: A comprehensive review on current therapies and oligonucleotide-based interventions under development. Pharmacol Res 2016;103:253-69.

38. Ishtiaq S, Edwards S, Sankaralingam A, et al. The effect of nitrogen containing bisphosphonates, zoledronate and alendronate, on the production of pro-angiogenic factors by osteoblastic cells. Cytokine 2015;71:154-60.

39. Nau C, Seebach C, Trumm A, et al. Alteration of Masquelet's induced membrane characteristics by different kinds of antibiotic enriched bone cement in a critical size defect model in the rat's femur. Injury 2016;47:325-34.

40. Ding XC, Ma WL, Li MK, et al. A Meta-analysis of the Value of $v W F$ in the Diagnosis of Liver Cirrhosis with Portal Hypertension. J Clin Transl Hepatol 2019;7:3-8.

41. Zuk J, Snarska-Drygalska A, Malinowski KP, et al. Unfavourably altered plasma clot properties in patients with primary Raynaud's phenomenon: association with venous thromboembolism. J Thromb Thrombolysis 2019; 47:248-54.

42. Olausson N, Mobarrez F, Wallen H, et al. Microparticles reveal cell activation during IVF - a possible early marker of a prothrombotic state during the first trimester. Thromb Haemost 2016;116:517-23. 